\title{
Probing the Earth's interior with a large-volume liquid scintillator detector
}

\author{
Kathrin A. Hochmuth, ${ }^{1,2, *}$ Franz v. Feilitzsch, ${ }^{1}$ Brian D. Fields, ${ }^{3}$ \\ Teresa Marrodán Undagoitia, ${ }^{1}$ Lothar Oberauer, ${ }^{1}$ \\ Walter Potzel, ${ }^{1}$ Georg G. Raffelt, ${ }^{2}$ and Michael Wurm ${ }^{1}$ \\ ${ }^{1}$ Technische Universität München, Physik Department E15, \\ James-Franck-Strasse, 85748 Garching, Germany \\ ${ }^{2}$ Max-Planck-Institut für Physik (Werner-Heisenberg-Institut), \\ Föhringer Ring 6, 80805 München, Germany \\ ${ }^{3}$ Center for Theoretical Astrophysics, Department of Astronomy, \\ University of Illinois, Urbana, IL 61801, USA
}

\begin{abstract}
A future large-volume liquid scintillator detector would provide a high-statistics measurement of terrestrial antineutrinos originating from $\beta$-decays of the uranium and thorium chains. In addition, the forward displacement of the neutron in the detection reaction $\bar{\nu}_{e}+p \rightarrow n+e^{+}$provides directional information. We investigate the requirements on such detectors to distinguish between certain geophysical models on the basis of the angular dependence of the geoneutrino flux. Our analysis is based on a Monte-Carlo simulation with different levels of light yield, considering both unloaded and gadolinium-loaded scintillators. We find that a $50 \mathrm{kt}$ detector such as the proposed LENA (Low Energy Neutrino Astronomy) will detect deviations from isotropy of the geoneutrino flux significantly. However, with an unloaded scintillator the time needed for a useful discrimination between different geophysical models is too large if one uses the directional information alone. A Gd-loaded scintillator improves the situation considerably, although a $50 \mathrm{kt}$ detector would still need several decades to distinguish between a geophysical reference model and one with a large neutrino source in the Earth's core. However, a high-statistics measurement of the total geoneutrino flux and its spectrum still provides an extremely useful glance at the Earth's interior.
\end{abstract}

*Electronic address: hochmuth@ph.tum.de 


\section{INTRODUCTION}

The first measurement of the $\bar{\nu}_{e}$ flux from natural radioactive elements in the Earth [1] has triggered a lot of excitement about the future of "neutrino geology" [2, 3, 4, ,5]. The geoneutrino ${ }^{1}$ flux could deliver new information about the interior of the Earth, in particular its radiochemical composition, and thus shed new light on the question of how the Earth and other planets formed. Such an ambitious programme requires detectors of the next generation that are able to provide much larger event rates.

One possible future detector that may well serve this purpose is LENA (Low Energy Neutrino Astronomy) that has been proposed by several of us [6] for high-statistics solar neutrino spectroscopy, for spectroscopy of the cosmic diffuse supernova neutrino background (DSNB), as a detector for the next galactic supernova, and to search for proton decay [7]. Present design studies for LENA assume $50 \mathrm{kt}$ of liquid scintillator that would provide a geoneutrino rate of roughly one thousand events per year from the dominant

$$
\bar{\nu}_{e}+p \rightarrow n+e^{+}
$$

inverse beta-decay reaction.

While liquid scintillator detectors do not provide direct angular information, indirectly one can retrieve directional information because the final-state neutron is displaced in the forward direction. The offset between the $e^{+}$and the neutron-capture locations can be reconstructed, although with large uncertainties. The CHOOZ reactor neutrino experiment has demonstrated a nontrivial directional sensitivity in that it was able to locate the reactor within $18^{\circ}$ half-cone aperture (68\% C.L.) on the basis of 2500 reconstructed events in a Gdloaded scintillator [8]. Therefore, it is natural to study the requirements for a future largevolume liquid scintillator detector to discriminate between different geophysical models of the Earth that differ both by their total neutrino fluxes and the neutrino angular distributions.

We will consider two types of detectors. Motivated by current design studies for LENA we will consider a $50 \mathrm{kt}$ detector using a PXE based scintillator. However, in this case it is difficult to locate the neutron-capture event on protons because a single $2.2 \mathrm{MeV}$ gamma is released that travels on average $22.4 \mathrm{~cm}$ before its first Compton interaction. Therefore, the

\footnotetext{
${ }^{1}$ It is understood that this term refers to electron-antineutrinos emitted by the Earth's natural radioactive elements.
} 
event reconstruction is relatively poor. As a second case we study a Gd-loaded scintillator where the neutron capture releases on average 3 photons with a total of $8 \mathrm{MeV}$, allowing for a much better event reconstruction. Moreover, the spatial resolution can likely be improved beyond the original $\mathrm{CHOOZ}$ experiment if one uses photomultipliers that are fast enough (time jitter $\sim 1 \mathrm{~ns}$ ) to use the photon arrival times for the event reconstruction.

For the geoneutrino flux we will consider a continental and an oceanic location. In each case we will use a reference model and exotic cases with an additional strong neutrino source in the Earth's core or with enhanced neutrino emission from the mantle.

We will begin in Sec. III with a discussion of the principle of geoneutrino detection in large-volume scintillator detectors as well as possible scintillator properties. In Sec. III we introduce our geophysical models. In Sec. IV we turn to the main part of our work, a MonteCarlo study of the discriminating power of different detectors between different geophysical models and conclude in Sec. V]

\section{GEONEUTRINO DETECTION}

\section{A. Directional information from neutron displacement}

In a scintillator detector, geoneutrinos are measured by the inverse beta-decay reaction Eq. (II) with an energy threshold of $1.8 \mathrm{MeV}$. The cross section is

$$
\sigma=9.52 \times 10^{-44} \mathrm{~cm}^{2} \frac{E_{+}}{\mathrm{MeV}} \frac{p_{+}}{\mathrm{MeV}}
$$

where $E_{+}$is the total energy of the positron and $p_{+}$its momentum. The visible energy $E_{\mathrm{vis}}=E_{+}+m_{e}$ always exceeds $1 \mathrm{MeV}$ because the positron annihilates with an electron of the target. By measuring the visible energy one can determine the neutrino energy as $E_{\nu} \approx E_{\mathrm{vis}}+0.8 \mathrm{MeV}$ because the kinetic energy of the neutron is typically around $10 \mathrm{keV}$ and thus negligible. After thermalization the neutron is captured by a nucleus, thus tagging the inverse beta decay reaction.

Kinematics implies that the neutron is scattered roughly in the forward direction with respect to the incoming neutrino [9], this being the key ingredient for obtaining directional information. The maximum scattering angle is

$$
\cos \theta_{\max }=\frac{\sqrt{2 E_{\nu} \Delta-\left(\Delta^{2}-m_{e}^{2}\right)}}{E_{\nu}},
$$


where $\Delta=m_{n}-m_{p}$ and $m_{n}, m_{p}$ and $m_{e}$ are the masses of the neutron, proton and positron, respectively. In this extreme case, the neutron and positron momenta are perpendicular to each other. For the maximum relevant geoneutrino energy of $3.2 \mathrm{MeV}$ one obtains $\cos \theta_{\max }=$ 0.79 , which is equivalent to $\theta_{\max }=37.8^{\circ}$. However, most geoneutrinos have energies much closer to threshold and the maximum angles are much closer to the forward direction. The average displacement between the neutron and positron events is then theoretically found to be about $1.7 \mathrm{~cm}[9]$.

The reactor experiment CHOOZ, using a Gd-loaded scintillator, has measured an average neutron displacement from the $e^{+}$event of $1.9 \pm 0.4 \mathrm{~cm}[8]$. However, once the neutron has been thermalized by collisions with protons, it diffuses some distance before being captured so that the actual displacement varies by a large amount for individual events. In a PXE based scintillator the average time interval until capture on a proton is $180 \mu \mathrm{s}$, leading to an uncertainty $\sigma$ of the displacement of about $4 \mathrm{~cm}$ for the $\mathrm{x}-, \mathrm{y}$ - and $\mathrm{z}$-direction [9]. With Gd loading $\sigma$ is reduced to approximately $2.4 \mathrm{~cm} \mathrm{[9]} \mathrm{because} \mathrm{the} \mathrm{neutron} \mathrm{diffusion} \mathrm{time} \mathrm{is}$ much shorter, on average about $30 \mu \mathrm{s}$ [8].

\section{B. PXE-based scintillator}

One option for the proposed LENA detector is to use a scintillator based on PXE (phenylo-xylylethane, $\mathrm{C}_{16} \mathrm{H}_{18}$ ). PXE has a high light yield, it is non hazardous, has a relatively high flashpoint of $145^{\circ} \mathrm{C}$, and a density of $0.985 \mathrm{~g} / \mathrm{cm}^{3}$ [10]. A possible admixture of dodecane $\left(\mathrm{C}_{12} \mathrm{H}_{26}\right)$ increases the number of free protons and improves the optical properties. A blend of $20 \%$ PXE and $80 \%$ dodecane shows a decrease in light yield of about $20 \%$ relative to pure PXE, an attenuation length of about $11 \mathrm{~m}$ and an increase in the number of free protons by $25 \%$ [1]].

In this paper we consider a detector with a total volume of about $70 \times 10^{3} \mathrm{~m}^{3}$. This could be realized with a cylindrical detector of $100 \mathrm{~m}$ length and $30 \mathrm{~m}$ diameter. An outer water Cherenkov detector with a width of $2 \mathrm{~m}$ acts as a muon veto. In order to shield against external gamma and neutron radiation a fiducial volume of about $42 \times 10^{3} \mathrm{~m}^{3}$ with a total number of $2.5 \times 10^{33}$ free protons as target can be realized using a scintillator mixture as mentioned above with 20\% PXE and 80\% dodecane. In Monte-Carlo calculations the light

yield of events in LENA has been determined [7]. For events in the central detector region 
the yield $N_{\mathrm{pe}}$, measured in photo-electrons (pe) per $\mathrm{MeV}$ energy deposition, can be expressed as $N_{\mathrm{pe}} \approx 400 \mathrm{pe} / \mathrm{MeV} \times c$, where $c$ is the optical coverage which depends on the number and aperture of the photomultiplier tubes (PMTs). A maximal coverage $c_{\max } \approx 0.75$ can not be exceeded so that we assume the maximal light yield to be around $300 \mathrm{pe} / \mathrm{MeV}$. For instance, the use of 12,000 PMTs with a diameter in aperture of $50 \mathrm{~cm}$ would result in an optical coverage of about $30 \%$ and a light yield $N_{\mathrm{pe}} \simeq 120 \mathrm{pe} / \mathrm{MeV}$. This can be obtained either by using PMTs like in the Super-Kamiokande experiment or by smaller PMTs equipped with light concentrators as they were developed for the Counting Test Facility (CTF) at the Gran Sasso underground laboratory [12]. For events off the axis of the cylinder the light yield would be enhanced. Hence, low-energy spectroscopy even in the sub-MeV region should be possible in LENA.

For a detection of the positron-neutron displacement the ability of the detector to locate the absorption position of both particles is crucial. The experimental reconstruction of both events is possible by analyzing the arrival times and the number of photons in each individual PMT. The position uncertainty depends on the total yield of registered photoelectrons. In the CTF, the measured position uncertainty was around $10 \mathrm{~cm}$ in each direction for events with 300 photo-electrons and it was shown that the uncertainty scales with the inverse square-root of that number [13]. Therefore, we will use a Gaussian distribution for the uncertainty of the positron event reconstruction with equal width in each direction of

$$
\sigma_{e^{+}}=10 \mathrm{~cm}\left(\frac{300 \mathrm{pe} / \mathrm{MeV}}{N_{\mathrm{pe}}} \frac{1 \mathrm{MeV}}{E_{\mathrm{vis}}}\right)^{1 / 2}
$$

where $N_{\text {pe }}$ is the light yield and $E_{\text {vis }}$ the visible energy released by the positron.

In PXE-based scintillators the neutron is captured by a proton with nearly $100 \%$ efficiency within an average time interval of about $180 \mu \mathrm{s}$, subsequently emitting a $2.2 \mathrm{MeV}$ gamma. This photon has a mean free path of $22.4 \mathrm{~cm}$ before its first Compton scattering so that the event reconstruction is much more uncertain than for the positron event. We have simulated this case by taking into account multiple Compton scatterings of the $2.2 \mathrm{MeV}$ gamma. The position of each gamma emission, representing the position of the neutron capture, is reconstructed by composing the energy-weighted sum of each Compton scattering event, taking into account the instrumental resolution. The distribution of the reconstructed

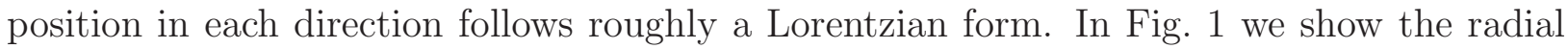
distribution of the reconstructed positions of these events for light yields of $N_{\mathrm{pe}}=50,300$ and 


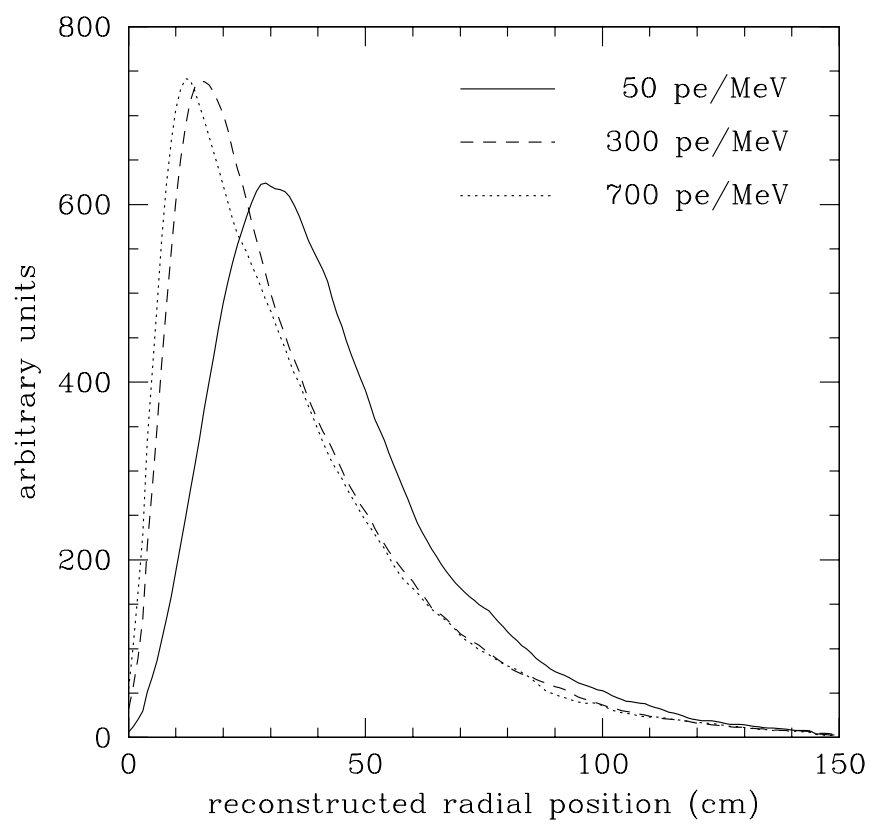

FIG. 1: Monte-Carlo simulation of the radial distribution of a $2.2 \mathrm{MeV} \gamma$-quantum in an unloaded PXE scintillator. The curves are for light yields of $N_{\mathrm{pe}}=50,300$ and $700 \mathrm{pe} / \mathrm{MeV}$ as indicated.

$700 \mathrm{pe} / \mathrm{MeV}$. Increasing the light yield does not significantly narrow the distribution because its width is dominated by the large Compton mean free path of the $2.2 \mathrm{MeV}$ photon. With reduced light yield the position of the maximum as well as the mean value of the distribution shifts towards larger values. This is caused by the increased uncertainty of the instrumental resolution.

\section{Gadolinium-loaded scintillator}

In PXE-based scintillators the neutron is captured by a proton with nearly $100 \%$ efficiency within an average time interval of about $180 \mu$ s, subsequently emitting a $2.2 \mathrm{MeV}$ gamma. However, one can also put an additive into the scintillator to enhance the delayed-neutron signal. Both lithium [14] and gadolinium 8] loaded scintillators have been used in this way for neutrino experiments or are planned in the future [15]. With a concentration of about $0.1 \%$ by mass, Gd captures neutrons with $90 \%$ efficiency and isotropically emits a total 
energy of about $8 \mathrm{MeV}$ in a gamma cascade with an average of 3 photons. Note however, that in a Gd-loaded PXE/dodecane scintillator the light yield is reduced by typically 10-20\% compared to an unloaded scintillator.

For Gd-loaded scintillators we use the measurements performed in the CHOOZ experiment. The neutron response of the detector was measured with a ${ }^{252} \mathrm{Cf}$ source and the position uncertainty of a neutron capture event was $19 \mathrm{~cm}$ at $1 \sigma$ in each direction [16]. The distribution was Gaussian and the light yield was measured to be $N_{\text {pe }}=(125 \pm 5)$ pe/MeV. The reconstruction was performed using only the information about the amplitude distribution of the PMTs. Based on these numbers we assume the position resolution of the neutron event for the LENA detector to be Gaussian with a width in each direction of

$$
\sigma_{n}=19 \mathrm{~cm}\left(\frac{125 \mathrm{pe} / \mathrm{MeV}}{N_{\mathrm{pe}}}\right)^{1 / 2} .
$$

In CHOOZ no information of the arrival time was used for the position reconstruction. However, the CTF measurements demonstrated that this yields the most valuable information, provided the time response of the PMTs is fast enough, i.e. a time jitter not much larger than 1 ns. Therefore, in a Gd-loaded scintillator one probably could achieve a much better neutron-event reconstruction so that our estimates are conservative.

\section{Backgrounds}

KamLAND has reported 152 events in the energy region relevant for geoneutrinos within a measuring time of 749 days and $3.5 \times 10^{31}$ target protons. From these events $127 \pm 13$ are due to background [17]. The most relevant background for the KamLAND site are reactor antineutrinos ( $80.4 \pm 7.2$ events). For the LENA detector positioned in the underground laboratory CUPP (Centre for Underground Physics in Pyhäsalmi) in Finland (longitude: $26^{\circ} 2.709^{\prime}$ E,latitude: $63^{\circ} 39.579^{\prime} \mathrm{N}, 1450 \mathrm{~m}$ of rock (4060 m.w.e.)) this background would be reduced by a factor $\simeq 12$, as the site is far away from reactors. Hence we expect for LENA at CUPP a reactor background rate of about 240 events per year in the relevant energy window from $1.8 \mathrm{MeV}$ to $3.2 \mathrm{MeV}$. This background can be subtracted statistically using the information on the entire reactor neutrino spectrum up to $\simeq 8 \mathrm{MeV}$.

Another important background for KamLAND is induced by radio impurities. A large

concentration of the long-lived isotope ${ }^{210} \mathrm{~Pb}$ is present in the KamLAND scintillator. In the 
decay chain of ${ }^{210} \mathrm{~Pb}$ the $\alpha$-emitting isotope ${ }^{210} \mathrm{Po}$ is present. Thus the reaction ${ }^{13} \mathrm{C}(\alpha, n){ }^{16} \mathrm{O}$ can occur, mimicking the signature of geoneutrinos due to neutron scattering on protons and the subsequent neutron capture. The number of these background events in KamLAND is estimated to be $42 \pm 11$ [17]. However, with an enhanced radiopurity of the scintillator, the background can be significantly reduced. Taking the radio purity levels of the CTF detector, where a ${ }^{210} \mathrm{Po}$ activity of $35 \pm 12 / \mathrm{m}^{3} \mathrm{~d}$ in PXE has been observed [10], this background would be reduced by a factor of about 150 compared to KamLAND and would account to less than 10 events per year in the LENA detector.

An additional background that imitates the geoneutrino signal is due to ${ }^{9} \mathrm{Li}$, which is produced by cosmic muons in spallation reactions with ${ }^{12} \mathrm{C}$ and decays in a $\beta$-neutron cascade. Only a small part of the ${ }^{9} \mathrm{Li}$ decays falls into the energy window which is relevant for geoneutrinos. KamLAND estimates this background to be $0.30 \pm 0.05$ [17]. At CUPP the muon reaction rate would be reduced by a factor $\simeq 10$ due to better shielding and this background rate should be at the negligible level of $\simeq 1$ event per year in LENA.

\section{MODELS OF THE EARTH}

A detailed density profile of the Earth, the Preliminary Reference Earth Model, was constructed by Dziewonski and Anderson in 1981 by monitoring seismic activities [18]. Based on the examination of meteorites and solar system materials (moon rocks and dust) and available Earth material, geologists have deduced a model for the distribution and concentration of elements in the Earth, the Bulk Silicate Earth Model [19]. The most dominant and abundant radioactive isotopes are ${ }^{238} \mathrm{U},{ }^{232} \mathrm{Th}$ and ${ }^{40} \mathrm{~K}$; their decays heat the Earth. The present-day total energy loss through the Earth's surface is about $40 \mathrm{TW}$ or $82 \mathrm{~mW} / \mathrm{m}^{2}$. The ratio of the energy production due to radioactivity to the total heat flow at the surface is known as the Urey ratio. In the Bulk Silicate Earth Model this ratio is assumed to be 0.5, attributing $20 \mathrm{TW}$ of the Earth's heat loss to radioactivity. Other estimates take the Urey ratio to be as large as 0.8 [20, 21].

The flux of geoneutrinos is directly linked to the rate of radioactive decays and to the generated heat. Therefore, it is of great interest to measure the geoneutrino flux and thus deduce the main contributor to the heat production. The elemental abundance ratios in the

Bulk Silicate Earth Model are $\mathrm{Th} / \mathrm{U} \approx 4$ and $\mathrm{K} / \mathrm{U} \approx 1.14 \times 10^{4}$. According to this model, 
radioactive isotopes are only in the crust and mantle because they are lithophile whereas the core is void of any significant amount of uranium, thorium or potassium. Different estimates for their abundances in the crust and mantle differ by factors of 2-3. An excellent overview of these abundances and their spread is given in the GERM Reservoir Data Base [22].

An Italian group of physicists has, in cooperation with geologists, constructed a reference model for the abundance values of uranium, thorium and potassium. They used the values referenced in GERM and derived a mean value for each element [23]. We will implement these abundances into our Reference Model of the geoneutrino angular distribution. For a discussion of the angular spectra of this model and its uncertainties see Ref. [4]. Our Reference Model is in accordance with the Bulk Silicate Earth constraint of a heat production of $20 \mathrm{TW}$ due to radioactive decays in the crust and mantle.

Other authors have speculated about the presence of uranium and thorium in the core, for example in the context of a putative georeactor [24, 25]. Moreover, the Earth's magnetic field is not yet fully understood, but seems to be generated by a complex interaction between core and mantle. The core itself is too hot to sustain a permanent magnetic field. Therefore, it is assumed that the magnetic field is powered by a geodynamo, where in a simplified picture the magnetic field is generated by the motion of the liquid outer core. One problem of this picture is the unknown energy source for the geodynamo. Powering it by energy from the inner core leads to cooling and solidification. This process is constrained by the present-day size of the inner core, implying that it must be younger than 1.7 Gyr [26] much less than the Earth's age of 4.5 Gyr. Therefore, several authors have concluded that there are radioactive elements in the core, providing heat and sustaining the geodynamo [26].

The geoneutrino flux depends sensitively on location. The oceanic crust is depleted in radioactive elements, whereas the flux on the continents is dominated by the crust. Thus an experiment situated in the Pacific Ocean, e.g. on Hawaii, would have better access to the oceanic crust and the mantle. For an experiment located on a continent we have assumed a thickness of $50 \mathrm{~km}$ for the crust, implying a total neutrino flux in our Reference Model of $4.2 \times 10^{6} \mathrm{~cm}^{-2} \mathrm{sec}^{-1}$ from uranium and $4.1 \times 10^{6} \mathrm{~cm}^{-2} \mathrm{sec}^{-1}$ from thorium decays. For an oceanic site we have chosen the crust to be rather thick $(50 \mathrm{~km})$, but not included any sediments. If one wanted to determine the mantle contribution, the oceanic crust would be a background to the measurement so that the assumption of a thick oceanic crust is conservative. The neutrino fluxes in this case are $1.25 \times 10^{6} \mathrm{~cm}^{-2} \mathrm{sec}^{-1}$ from uranium and 
TABLE I: Annual event rates for $2.5 \times 10^{33}$ target protons. Flavor oscillations have been included with a global reduction factor of 0.57 .

\begin{tabular}{lll}
\hline \hline Model & Continental Crust & Oceanic Crust \\
\hline Reference Model & $1.02 \times 10^{3}$ & $0.29 \times 10^{3}$ \\
(A) $20 \mathrm{TW}$ core & $1.35 \times 10^{3}$ & $0.62 \times 10^{3}$ \\
(B) $10 \mathrm{TW}$ core & $1.19 \times 10^{3}$ & $0.45 \times 10^{3}$ \\
(C) $20 \mathrm{TW}$ Lower Mantle & $1.44 \times 10^{3}$ & $0.71 \times 10^{3}$ \\
\hline \hline
\end{tabular}

$0.88 \times 10^{6} \mathrm{~cm}^{-2} \mathrm{sec}^{-1}$ from thorium decays. The zenith-angle distributions of the reference geoneutrino flux corresponding to continental and oceanic locations are shown in Fig. 2. Our assumption of a uniform oceanic crust with a thickness of $50 \mathrm{~km}$ has been made for computational reasons. Still, this model for the Hawaiian detector site is valid as our event rate is slightly lower than the rate found in [23]. In addition, as will become obvious in Sec. IV changes in the crustal thickness of an oceanic site by even an order of magnitude can not be resolved by the detector.

Besides our reference model we consider three "exotic" cases A, B and C, each of them either with a continental or an oceanic crust.

(A) Fully radiogenic model with additional uranium and thorium in the core, accounting for $20 \mathrm{TW}$ additional heat production. (Integrated neutrino flux increase of about $32 \%$ relative to the reference model in a continental location, and $116 \%$ in an oceanic location.)

(B) Same with 10 TW in the core. (Flux increase of $16 \%$ and $58 \%$, respectively.)

(C) $20 \mathrm{TW}$ in the Lower Mantle. (Flux increase of $41 \%$ and $148 \%$, respectively.)

The zenith-angle distributions of the neutrino fluxes from these models have been determined along the lines of Ref. [4] and is shown in Fig. 2]

To obtain the event rate in a scintillator detector, neutrino flavor oscillations have to be accounted for by including a global $\bar{\nu}_{e}$ survival-probability factor of 0.57 as measured by KamLAND [17]. Matter effects for oscillations are not important because of the small geoneutrino energies. Moreover, for geoneutrino energies of $1.8-3.2 \mathrm{MeV}$ and $\Delta m^{2}=7.9 \times 10^{-5} \mathrm{eV}^{2}$ the 

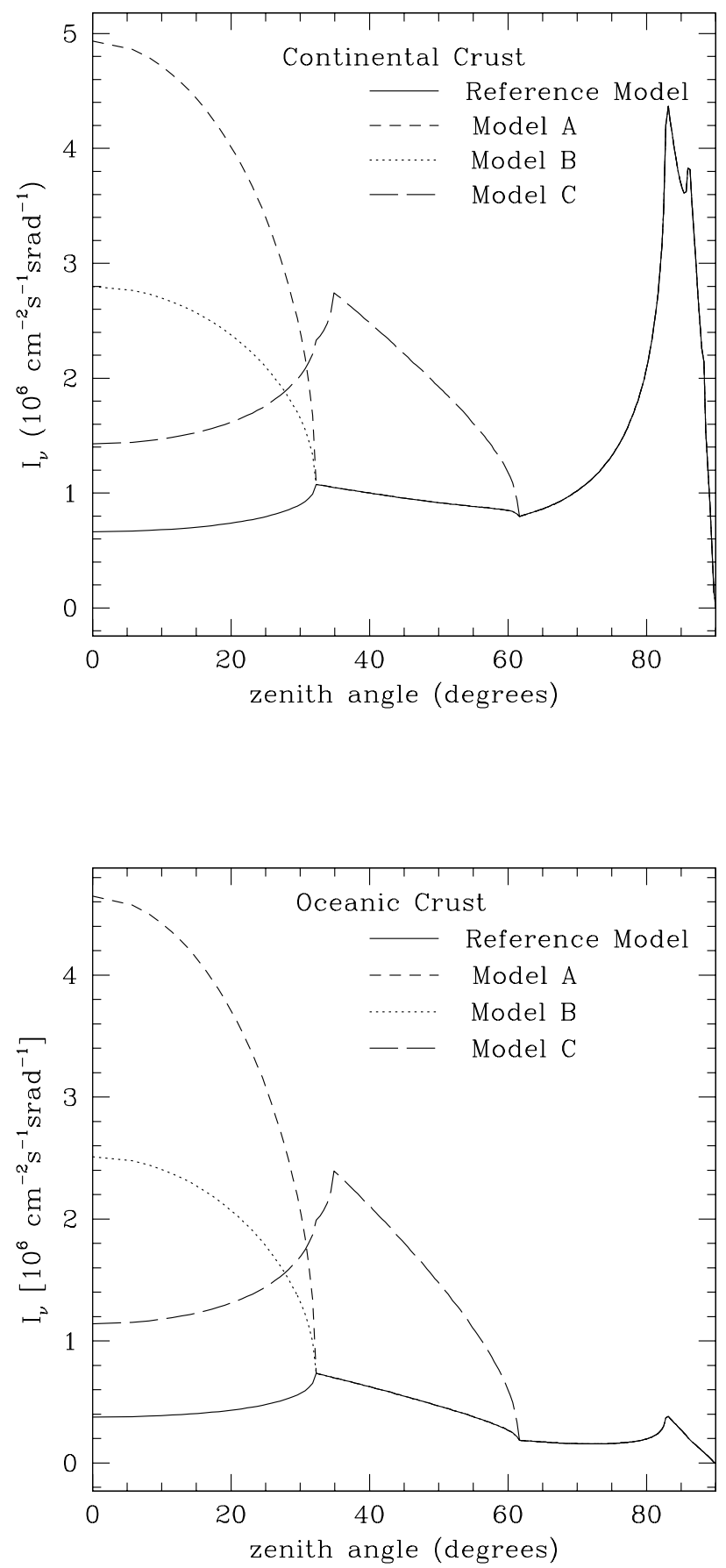

FIG. 2: Zenith-angle distribution of the geoneutrino flux where $\theta=0$ corresponds to the vertical direction. Shown is the total flux without flavor oscillations. Upper panel: Continental crust. Lower panel: Oceanic crust. 
vacuum oscillation length is $57-101 \mathrm{~km}$. Including distance and energy dependent survival probabilities is a negligible correction to a global reduction factor [27]. The annual event rates corresponding to our models, including the reduction factor, are shown in Tab. \for a 50 kton detector with a fiducial volume corresponding to $2.5 \times 10^{33}$ protons.

Up to now we have assumed that the exotic heat source in the Earth's core is caused by uranium and thorium decays, i.e. the neutrino spectrum from this additional source was taken to be identical with the geoneutrino spectrum from the crust and mantle. However, the possibility of a natural reactor in the Earth's core ("georeactor") has been discussed

in the literature [24, 25]. In this case the neutrino flux could be similar to that from an ordinary power reactor with energies reaching up to about $8 \mathrm{MeV}$. With this assumption the total $4 \pi$-georeactor neutrino flux can be estimated to be $\Phi_{\nu} \simeq 1.9 \times 10^{23} \mathrm{~s}^{-1}$ for a thermal power of 1 TW. Taking into account neutrino oscillations, the distance to the center of the Earth, and the detection cross section we calculate an event rate of about $210 \mathrm{y}^{-1} \mathrm{TW}^{-1}$ in LENA. At Pyhäsalmi one would observe about 2200 events per year due to neutrinos from nuclear power plants. Assuming a systematic uncertainty for the neutrino flux from the power plants of $6.5 \%$, as suggested in 17], we conclude that LENA will be able to identify a georeactor of $\geq 2 \mathrm{TW}$ after one year of measurement with a $3 \sigma$ significance. The influence of the uncertainty of the mixing angle $\theta_{12}$ is negligible, because the flux of both the georeactor and the power plants depends on $\theta_{12}$ in the same way.

\section{MONTE-CARLO STUDY}

To study the power of directional discrimination of a large liquid-scintillator detector we have performed a Monte-Carlo simulation of a large number of geoneutrino events and the corresponding directional reconstruction. We have assumed that the detector response is independent of the event location, i.e. only the spatial separation between the event $\bar{\nu}_{e}+p \rightarrow n+e^{+}$and the location of neutron capture is relevant. However, as pointed out in chapter II, we consider a position resolution of point like events located at the central axis of the detector. As the light yield and hence also the position resolution increases for offaxis events our assumption is conservative. We have assumed that, on average, the neutron capture point is displaced by $1.9 \mathrm{~cm}$ in the forward direction relative to the $e^{+}$event in agreement with the CHOOZ measurement [16]. Moreover, we have assumed that neutron 

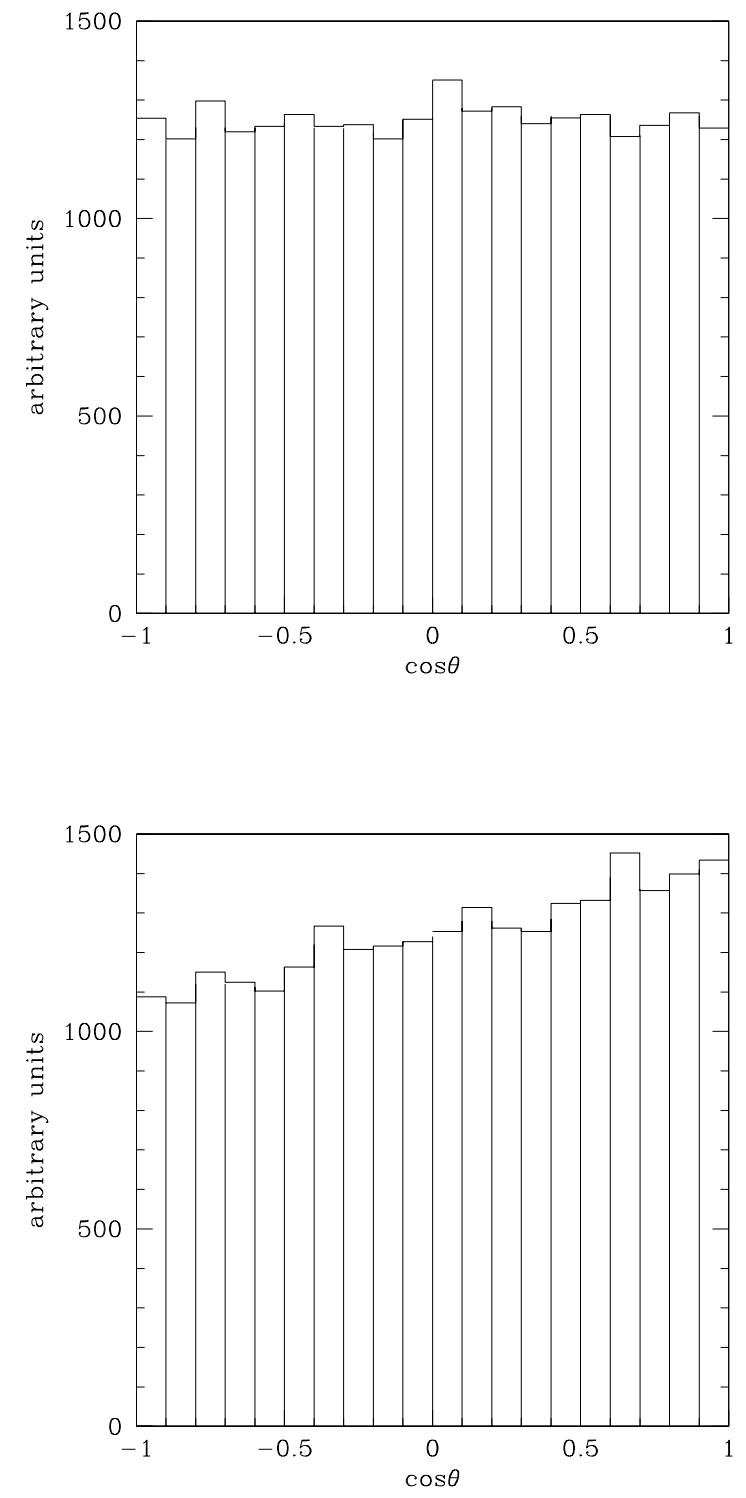

FIG. 3: Monte-Carlo example of reconstructed zenith-angle distribution of 25,000 events in a Gdloaded detector. Upper panel: Neutrinos injected from the horizontal direction $(\cos \theta=0)$. Lower panel: Neutrinos injected from the vertical direction $(\cos \theta=1)$.

diffusion before capture causes a Gaussian distribution around this mean value with a width $\sigma_{x}=\sigma_{y}=\sigma_{z}=4.0 \mathrm{~cm}$ for an unloaded PXE-based scintillator, whereas $2.4 \mathrm{~cm}$ is taken for a Gd-loaded scintillator as described in Sec. III

In addition to this distribution, the main uncertainty originates from the reconstruction 
of both events. For the positron event we have assumed that the reconstructed location follows a Gaussian distribution with a width given by Eq. (4). The actual spread of relevant visible energies is small so that we have always used $E_{\text {vis }}=1.4 \mathrm{MeV}$ as a typical value. For an unloaded scintillator, the reconstruction of the neutron event introduces an even larger uncertainty; we have used a distribution as in Fig. 1 appropriate for the given light yield. For a Gd-loaded scintillator the uncertainty can be well fitted by a Gaussian distribution with a width given by Eq. (41).

For the sake of illustration we discuss the reconstructed zenith-angle distributions for the two extreme cases where all neutrinos come from the horizontal direction or all of them come vertically from below, i.e with $\cos \theta=1$. Figure 3 displays the reconstructed zenithangle distribution of both cases, each generated with 25,000 neutrino events and using a Gd-loaded detector with a light yield $N_{\text {pe }}=300 \mathrm{pe} / \mathrm{MeV}$. We conclude that, given the relatively poor angular reconstruction capability of scintillator detectors, the only angulardistribution information that can be extracted is the slope of the distributions shown in Fig. 3. Put another way, one can extract the total event rate and the dipole contribution of the angular distribution, whereas a determination of higher multipoles is unrealistic. Therefore, we write the reconstructed zenith-angle distribution in the form

$$
\frac{d \dot{N}}{d \cos \theta}=\dot{N}\left(\frac{1}{2}+p \cos \theta\right)
$$

where the event rate $\dot{N}$ and the coefficient $p$ are the two numbers that characterize a given configuration of geophysical model and detector type.

The event rates for our fiducial detector size with $2.5 \times 10^{33}$ target protons and different geophysical models have already been reported in Tab. [1] What remains to be determined by means of a Monte-Carlo simulation are the corresponding coefficients $p$ and their uncertainty. In Tab. II we show the results for $p$ for different cases, always assuming a light yield of $300 \mathrm{pe} / \mathrm{MeV}$. The uncertainty $\sigma_{p}$ of the measured $p$ value scales with the inverse square root of the number of events $N$ so that $s_{p}=\sigma_{p} \sqrt{N}$ is a quantity independent of $N$. The value of $s_{p}$ can be derived analytically for $p=0$, yielding

$$
s_{p}=\frac{\sqrt{3}}{2}=0.866
$$

which is valid for all $p \ll 1$. We have checked with our Monte Carlo that Eq. (17) indeed applies to all $p$ values of interest to us. 
TABLE II: Coefficient $p$ for the reconstructed zenith-angle distribution for different Earth models and different detector types, always assuming a light yield of $300 \mathrm{pe} / \mathrm{MeV}$.

\begin{tabular}{lll}
\hline \hline Model & \multicolumn{2}{c}{ Coefficient $p$ for scintillator detectors } \\
& Unloaded PXE & Gd-loaded \\
\hline Continenal Crust & & \\
Reference Model & 0.0283 & 0.0377 \\
(A) 20 TW core & 0.0377 & 0.0521 \\
(B) 10 TW core & 0.0333 & 0.0459 \\
(C) 20 TW Lower Mantle & 0.0351 & 0.0485 \\
Oceanic Crust & & \\
Reference Model & 0.0468 & 0.0646 \\
(A) 20 TW core & 0.0597 & 0.0824 \\
(B) 10 TW core & 0.0560 & 0.0772 \\
\hline \hline
\end{tabular}

The number of events it takes to distinguish at the $1 \sigma$ level between an isotropic event distribution $(p=0)$ and the actual coefficient is given by $N_{1 \sigma}=\left(s_{p} / p\right)^{2}=(3 / 4) p^{-2}$. For our reference model at a continental site we find $N_{1 \sigma} \approx 500$ events, for an oceanic site about 200 events. In order to distinguish a geophysical model $i$ from model $j$ at the $1 \sigma$ level, the required number of events is

$$
N_{1 \sigma}=\frac{2 s_{p}^{2}}{\left(p_{i}-p_{j}\right)^{2}}=\frac{3}{2} \frac{1}{\left(p_{i}-p_{j}\right)^{2}} .
$$

A detection at the $n \sigma$ level requires $n^{2}$ times more events.

In the same way as for Tab. II] we have calculated the slope $p$ for different light yields of the scintillator and have determined the number of events it takes to distinguish each of the exotic models from the reference case. In Fig. [4 we display $N_{1 \sigma}$ for these cases and the continental-crust situation as a function of the light yield $N_{\text {pe }}$, both for an unloaded PXE-type detector and a Gd-loaded one. In Fig. 5 we show the same for the oceanic crust. In the oceanic location we do not show model $\mathrm{C}$ because it corresponds to an increased flux from the mantle, i.e. it is essentially identical with the reference model except for the total flux and thus can not be distinguished on the basis of the zenith-angle distribution.

Of course, the time required to achieve this discriminating power depends on the detector 
size. For our fiducial volume with $2.5 \times 10^{33}$ target protons as in LENA one needs to scale with the event rates shown in Tab. I. In a continental-crust location, all models produce an event rate of roughly 1000 events per year, in full agreement with the KamLAND measurement [17]. Assuming for example a realistic light yield of $120 \mathrm{pe} / \mathrm{MeV}$ for the LENA detector (unloaded) one would have to measure roughly 150 year to distinguish even our most optimistic model A from the reference model. Therefore, even with optimistic assumptions a $50 \mathrm{kt}$ detector would need several decades for distinguishing in a meaningful way between different geophysical models on the basis of the angular event distribution. Moreover, detector backgrounds should be included in a realistic assessment.

\section{CONCLUSIONS}

A future large-volume scintillator detector such as the proposed $50 \mathrm{kt}$ LENA would provide a high-statistics measurement of the geoneutrino flux. The event rate would depend strongly on the detector location, notably on whether an oceanic site such as Hawaii is chosen where a reference event rate of about 300 per year (50 kt scintillator) is expected or a continental site such as the Pyhäsalmi mine in Finland where the reference rate would be about 1000 per year. Therefore, the geoneutrino flux could be measured with high significance and would allow one to distinguish between different geophysical models.

The forward displacement of the neutron in the inverse beta decay detection reaction provides directional information on the geoneutrino flux. We have studied if this effect can be used to distinguish between different geophysical models, notably if one could diagnose a strong exotic energy source in the Earth's core under the assumption that its neutrino spectrum is identical with that emitted by the crust and mantle. While a deviation from an isotropic flux can be ascertained with high significance, we find that a $50 \mathrm{kt}$ detector is too small to distinguish between different geophysical models on the basis of the directional information alone, except perhaps for extreme cases and optimistic assumptions about the detector performance.

In our study we have only used the neutrino flux from the Earth, ignoring the contribution from power reactors because it depends strongly on location. For example, in Pyhäsalmi the neutrino flux from power reactors adds roughly $25 \%$ to the counting rate in the energy window relevant for geoneutrinos. This contribution is not negligible, but it does not change 

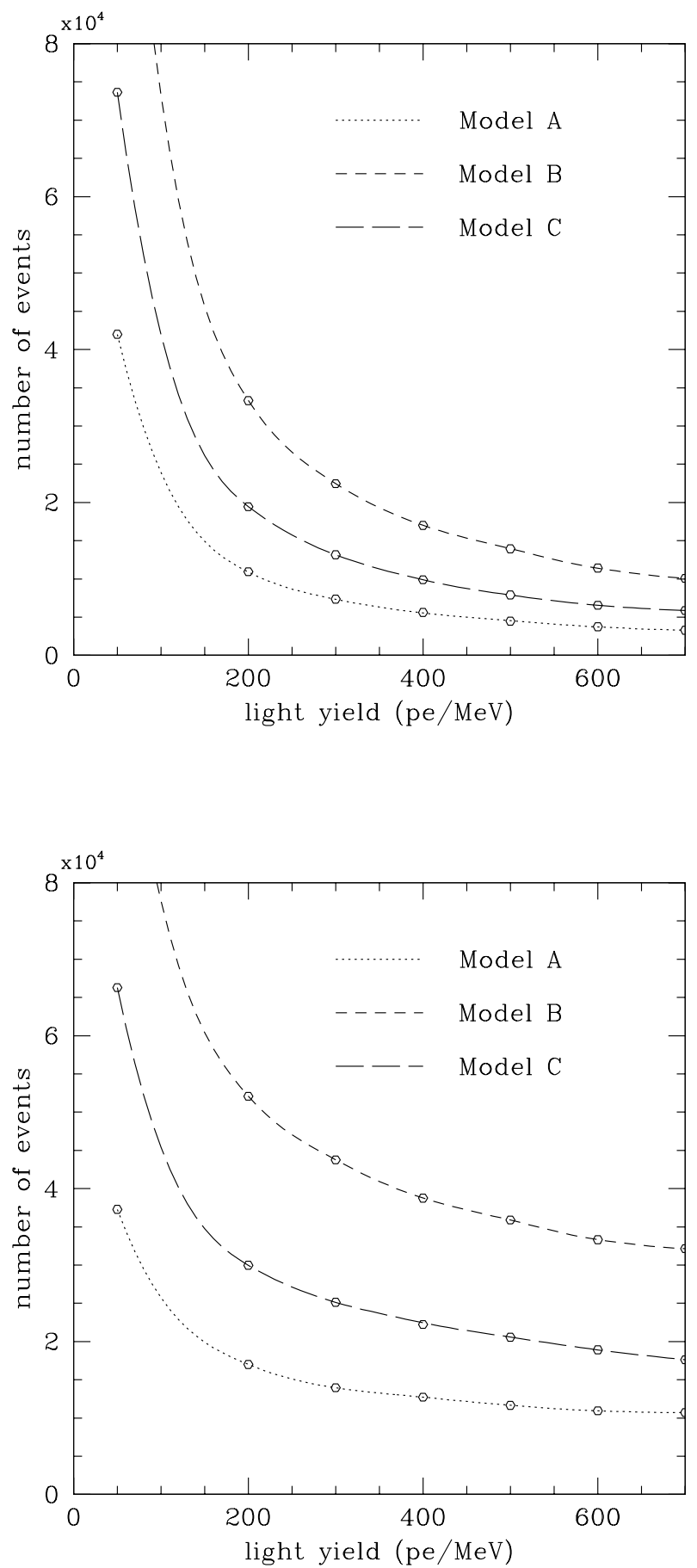

FIG. 4: Number of events needed to distinguish between models A, B or C and the continentalcrust reference model at $1 \sigma$ significance. The points correspond to the values calculated with the Monte Carlo. Upper panel: Gd-loaded scintillator. Lower panel: Unloaded PXE-type scintillator. 

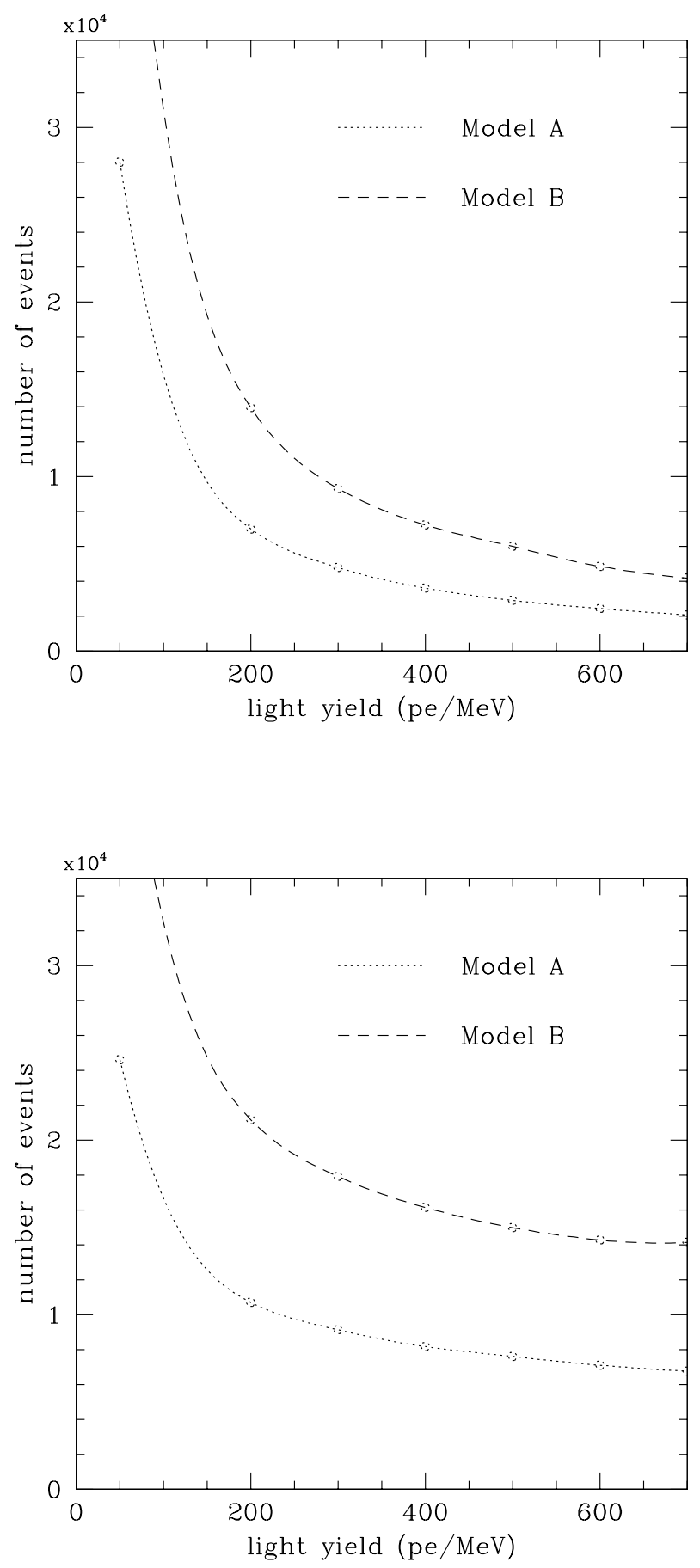

FIG. 5: Same as Fig. 4 for oceanic crust. Model C was not included because the neutrino flux has almost the same angular distribution as the reference model so that almost infinitely many events are needed for a discrimination. 
our overall conclusions.

We have also estimated the sensitivity of a LENA type detector for determining a hypothetical georeactor in the Earth's core. As a possible location the CUPP underground laboratory in Pyhäsalmi (Finland) was chosen and the background due to nuclear power plants was calculated. At CUPP a 2 TW georeactor could be identified at a statistical level of $3 \sigma$ after only one year of measurement.

In summary, large-volume scintillator detectors of the next generation will be extremely useful to study the interior of the Earth in the "light of neutrinos". However, the prime information will be the total geoneutrino flux and its spectrum. It would be extremely challenging to use the directional information alone to distinguish between different geophysical models.

\section{Acknowledgments}

We thank E. Lisi for crucial discussions of an earlier version of this paper. Partial support by the Maier-Leibnitz-Laboratorium (Garching), the Virtual Institute for Dark Matter and Neutrinos (VIDMAN, HGF), the Deutsche Forschungsgemeinschaft under Grant No. SFB375 and the European Union under the ILIAS project, contract No. RII3-CT-2004-506222, is acknowledged.

[1] T. Araki et al., "Experimental investigation of geologically produced antineutrinos with KamLAND," Nature 436 (2005) 499.

[2] G. Fiorentini, M. Lissia, F. Mantovani and R. Vannucci, "Geo-neutrinos: A new probe of earth's interior," Earth Planet. Sci. Lett. 238 (2005) 235 arXiv:physics/0508019.

[3] G. Fiorentini, M. Lissia, F. Mantovani and R. Vannucci, "How much uranium is in the Earth? Predictions for geo-neutrinos at KamLAND," Phys. Rev. D 72 (2005) 033017 hep-ph/0501111.

[4] B. D. Fields and K. A. Hochmuth, "Imaging the Earth's interior: The angular distribution of terrestrial neutrinos," hep-ph/0406001, accepted for publication in Earth, Moon and Planets 
[5] R. J. de Meijer, E. R. van der Graaf and K. P. Jungmann, "Quest for the nuclear georeactor," Nucl. Phys. News 14 (2004) 20 physics/0404046.

[6] L. Oberauer, F. von Feilitzsch and W. Potzel, "A large liquid scintillator detector for lowenergy neutrino astronomy," Nucl. Phys. Proc. Suppl. 138 (2005) 108.

[7] T. Marrodán Undagoitia et al., "Search for the proton decay $p \rightarrow K^{+} \bar{\nu}$ in the large liquid scintillator low energy neutrino astronomy detector LENA," Phys. Rev. D 72 (2005) 075014 hep-ph/0511230.

[8] M. Apollonio et al. [CHOOZ Collaboration], "Determination of neutrino incoming direction in the CHOOZ experiment and its application to supernova explosion location by scintillator detectors," Phys. Rev. D 61 (2000) 012001 hep-ex/9906011.

[9] P. Vogel and J. F. Beacom, "Angular distribution of neutron inverse beta decay, $\bar{\nu}_{e}+p \rightarrow$ $e^{+}+n, "$ Phys. Rev. D 60 (1999) 053003 hep-ph/9903554.

[10] H. O. Back et al. [Borexino Collaboration], "Phenylxylylethane (PXE): A high-density, highflashpoint organic liquid scintillator for applications in low-energy particle and astrophysics experiments", arXiv:physics/0408032.

[11] M. Wurm, "Untersuchungen zu den optischen Eigenschaften eines Flüssigszintillators und zum Nachweis von Supernovae Relic Neutrinos mit LENA", Diploma thesis, TU München, Germany (2005).

[12] L. Oberauer, C. Grieb, F. von Feilitzsch, I. Manno, "Production of light concentrators for BOREXINO and its Counting Test Facility", Nucl. Instrum. Meth. A 530 (2004) 453.

[13] G. Alimonti et al. [Borexino Collaboration], "A large-scale low-background liquid scintillation detector: The Counting Test Facility at Gran Sasso", Nucl. Instrum. Meth. A 406 (1998) 411.

[14] J. Hejwowski and A. Szymanski, "Lithium loaded liquid scintillator," Review of Scientific Instruments 32 (1961) 1057-1058.

[15] F. Ardellier et al., "Letter of intent for double-CHOOZ: A search for the mixing angle theta(13)," hep-ex/0405032,

[16] M. Apollonio et al., "Search for neutrino oscillations on a long base-line at the CHOOZ nuclear power station," Eur. Phys. J. C 27(2003) 331 hep-ex/0301017.

[17] T. Araki et al. [KamLAND Collaboration], "Measurement of neutrino oscillation with KamLAND: Evidence of spectral distortion," Phys. Rev. Lett. 94 (2005) 081801 hep-ex/0406035.

[18] A. M. Dziewonski and D. L. Anderson, "Preliminary Reference Earth Model," Phys. Earth 
Planet. Interiors 25 (1981) 297. For a tabulation see http://solid_Earth.ou.edu/prem.html

[19] W. F. McDonough and S.-S. Sun, "The composition of the Earth," Chem. Geol. 120 (1995) 223.

[20] C. Stein, in: Global Earth Physics: A Handbook of Physical Constants, AGU Reference Shelf 1, edited by T. J. Ahrens (American Geophysical Union, Washington, 1995), p. 144.

[21] D. McKenzie and F. Richter, J. Geophys. Res. 86 (1981) 11667.

[22] Earth Reference Data and Models, http://earthref.org

[23] F. Mantovani, L. Carmignani, G. Fiorentini and M. Lissia, "Antineutrinos from the Earth: The reference model and its uncertainties," Phys. Rev. D 69 (2004) 013001 hep-ph/0309013.

[24] J. M. Herndon, J. Geomagn. Geoelectr. 45 (1993) 423.

[25] J. M. Herndon, "Nuclear georeactor origin of oceanic basalt He-3/He-4, evidence and implications," PNAS, March 18 (2003), Vol. 100, No. 6, pp. 3047-3050

[26] S. Labrosse et al., Earth Planet. Sci. Lett. 190 (2001) 111.

[27] G. Fiorentini, T. Lasserre, M. Lissia, B. Ricci and S. Schönert, "KamLAND, terrestrial heat sources and neutrino oscillations," Phys. Lett. B 558 (2003) 15 hep-ph/0301042. 